\title{
Solar storms, cycles and topology
}

\author{
H. Lundstedt ${ }^{\mathrm{a}}$ \\ Swedish Institute of Space Physics, Lund, Sweden
}

\begin{abstract}
Solar storms are produced due to plasma processes inside and between coronal loops. These loops are topologically examined using knot and braid theory. Solar cycles are topologically explored with a complex generalization of the three ordinary differential equations studied by Lorenz. By studying the Poincaré map we give numerical evidence that the flow has an attractor with fractal structure. The period is defined as the time needed for a point on a hyperplane to return to the hyperplane again. The periods are distributed in an interval. For large values of the Dynamo number there is a long tail toward long periods and other interesting comet-like features. We also found a relationship between the intensity of a cycle and the length for the previous cycle. Maunder like minima are also appearing. These general relations found for periods can further be physically interpreted with improved helioseismic estimates of the parameters used by the dynamical systems. Solar Dynamic Observatory is expected to offer such improved measurements.
\end{abstract}

\section{Introduction}

Geometrical figures make us understand. Figures can be observable 3D plasma loops, such as building blocks of solar storms (solar flares and coronal mass ejections). Mathematical topological descriptions can then be used to explore the state and the solar activity. However, figures can also be highly abstract and of dimension greater three. It can e.g. be the state space of the solar cycle evolution. Mathematical topological description can help us understand the evolution.

Today a differentiable manifold is a modern representation of a figure as a geometric object.

A topological space is a figure in the broadest sense. The space is described by a set. The structure is then described by the topology.

A differential manifold is a topological space such that any point on it has a neighborhood whose points can be described by local coordinates consisting of $n$ independent parameters, and the relationship among different local coordinates can be described by differentiable functions.

Differential forms [7] express various geometric structures of manifolds.

\section{Observations}

\subsection{Building blocks of solar storms}

Plasma loops play the role of building blocks of solar storms.

Figure 1 shows plasma loops observed in $171 \AA$, i.e in quiet corona and upper transition region, by Atmospheric Imaging Assembly (AIA) onboard Solar Dynamics Observatory (SDO). Different wavelengths make different plasma loops visible due to different temperature, density and instrument characteristics. AIA's 6 Fe-line EUV channels are assumed to cover the coronal thermal structure.

\footnotetext{
a e-mail: henrik@lund.irf.se
}

This is an Open Access article distributed under the terms of the Creative Commons Attribution-Noncommercial License 3.0, which permits unrestricted use, distribution, and reproduction in any noncommercial medium, provided the original work is properly cited. 


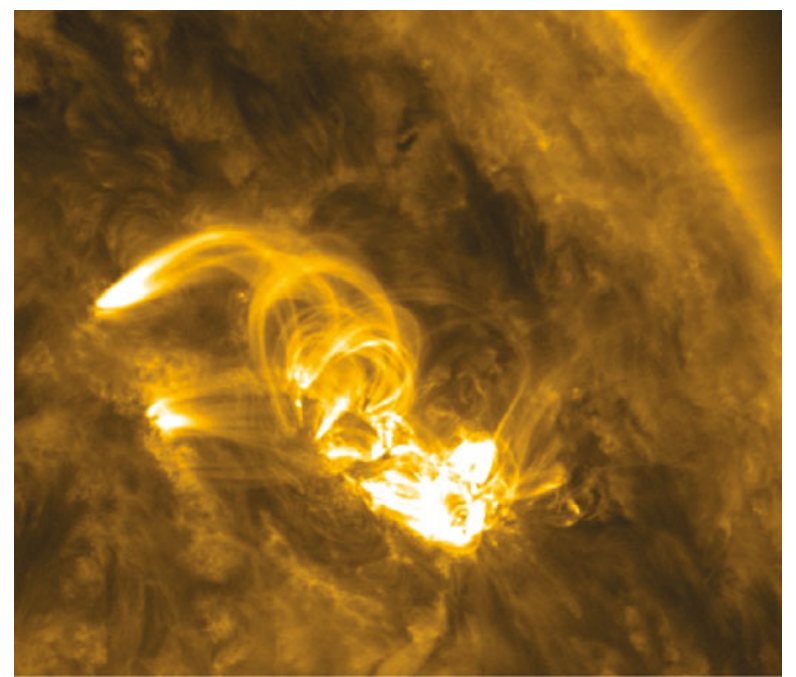

Fig. 1. Plasma loops observed in wavelength 171 Å with AIA onboard SDO on 12th June 2010.

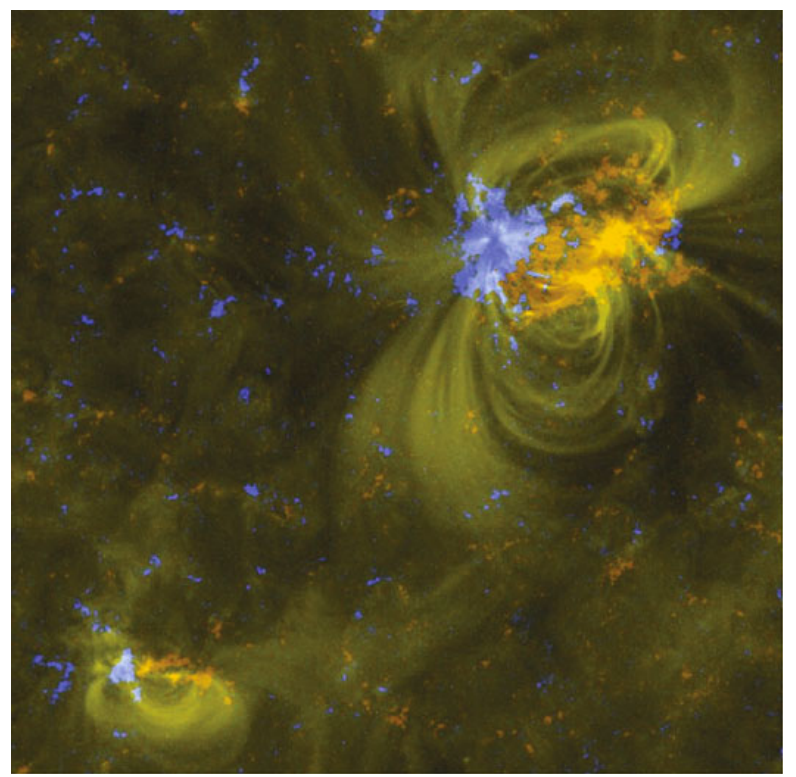

Fig. 2. Plasma loops observed in $171 \AA$ with AIA onboard SDO on 2nd June 2010. The longitudinal magnetic field observed with HMI onboard SDO is overlayed.

Figure 2 shows AR 11076 region in $171 \AA$ on 2nd June 2010. Overlayed is also the longitudinal magnetic field observed with Helioseismic and Magnetic Imager (HMI) onboard SDO.

Figure 3 shows how a prominence is becoming more and more twisted, kinked and then finally is lifting as a CME. In ideal conditions the helicity is conserved. Some twist can therefore be converted into writh. However, not too much before a kink instability starts. It has been suggested that CMEs are the way the Sun gets rid of its extra helicity [13].

\subsection{Indicators of solar cycles}

Observations of solar magnetic fields are only available for about five solar cycles. Further back in time only indicators of solar cycle activity are available. In Fig. 4 the sunspot number and ${ }^{14} C$ are plotted. 


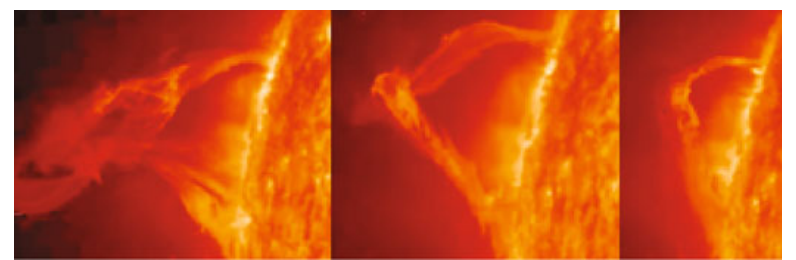

Fig. 3. Eruption of a prominence and CME observed on May 2010 in $304 \AA$ with AIA onboard SDO.
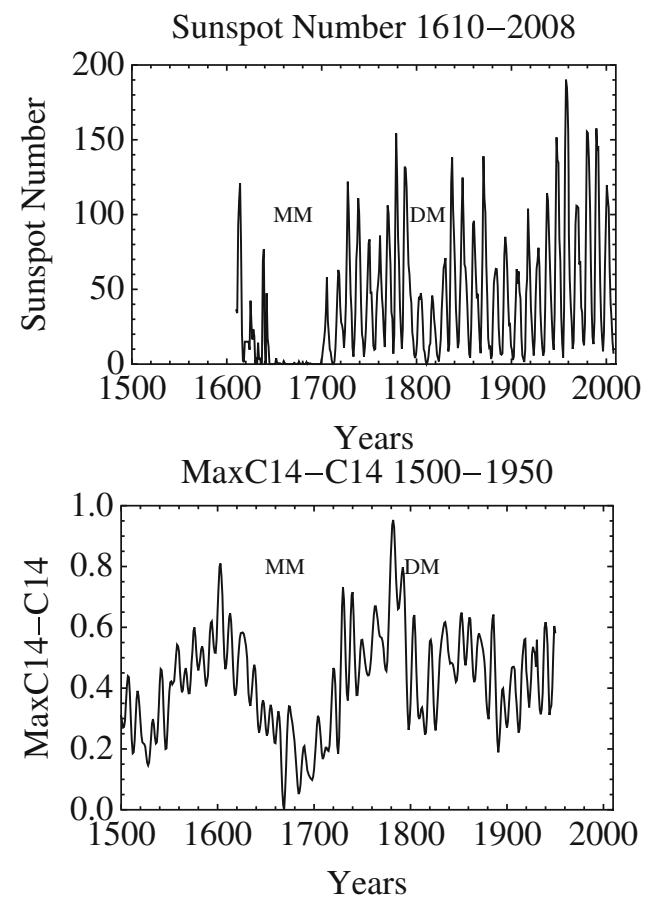

Fig. 4. Upper panel shows sunspot number. Lower panel shows ${ }^{14} C$. MM stands for Maunder Minimum and DM for Dalton Minimum.

The sunspot number is a coarse indicator of the variation of the toroidal magnetic field of the sun. During the Maunder minimum (MM) (1645-1715) very few sunspots appeared and no cyclicity is visible. The cycle length was also increasing just before the start of the MM ([8]). However, using ${ }^{14} C$ as and ${ }^{10} \mathrm{Be}$ indicator, cyclicity and periods are found during the MM $[1,12,14]$ and [16].

In Fig. 5 the period of a sunspot cycle vs. the following is plotted. There might be structures; long periods can be followed by a short or an averaged one and a short can be followed by a long one. However, the number of estimated solar cycle periods, is very small. Some of the estimates are also uncertain. The estimates were produced by National Geophysical Data Center (NGDC) in Boulder, USA.

\section{Solar storms and topology}

Coronal activity is looked upon as a response to activity below and under the solar surface. The energy to be released at times of solar storms (solar flares and CMEs) is assumed to be currents dissipated in plasma loops through various plasma instabilities such as kink instabilities. The most severe space weather effects are caused by severe solar storms, and especially fast halo CMEs.

Let us now discuss how plasma loops, 3D structures (Figs. 1-3), i.e the building blocks of solar storms, can be modelled with knots and braids. 


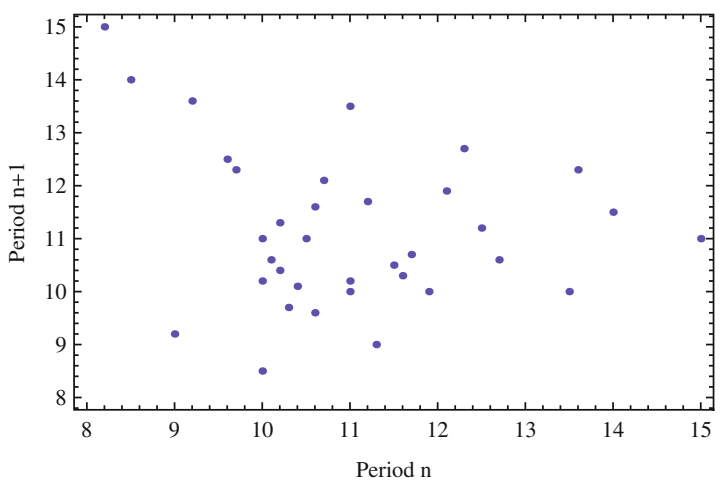

Fig. 5. Period $n$ vs. $n+1$ based on sunspot numbers.

\subsection{Knots, braids, linking number and helicity}

Knots are defined as closed loops embedded in ordinary 3-D space. A link is a collection of several such loops. Gauss found the topological invariant, the linking number, in trying to find asteroids [9]. The pair-wise linking is given by the Gauss integral,

$$
\mathcal{L}_{i j}=\frac{1}{4 \pi} \oint \oint d \mathbf{r}_{i} \times d \mathbf{r}_{j} \cdot \frac{\mathbf{r}_{i}-\mathbf{r}_{j}}{\left|\mathbf{r}_{i}-\mathbf{r}_{j}\right|^{3}} .
$$

The linking number of two loops (curves) can easily be calculated making use of the fact that knots can be represented by plane diagrams. These projections of the knots [18] onto a plane preserves crossing information. The linking number is then half the sum of the signed crossings.

$$
L\left(C_{1}, C_{2}\right)=\frac{1}{2} \sum_{i} \sigma_{i}\left(C_{1}, C_{2}\right)
$$

where $\sigma_{i}\left(C_{1}, C_{2}\right)=\sigma_{i}\left(C_{2}, C_{1}\right)= \pm 1$ is assigned to each crossings.

Braids are defined as systems of curves, which start from a line of equally spaced points and end at a parallel line, but winding round each other on the way. A braid word describes the successive crossings of a set of strings winding about each other between two parallel planes. An Artin presentation of 3-braids is given by,

$$
\left\{\sigma_{1}, \sigma_{2} \mid \sigma_{1} \sigma_{2} \sigma_{1}=\sigma_{2} \sigma_{1} \sigma_{2}\right\}
$$

The length of an Artin word equals the number of crossings seen in a braid diagram. Minimum crossings number provide a measure of complexity for braids. The energy of braided magnetic tubes has been estimated using minimum word length [3].

The Gauss linking number vanish for a Borromean ring knot (Fig. 6). Higher order invariants are therefore required to be able to model fine structures of plasma loops and more complex links. Such invariants have also been found based on differential forms, algebraic topology and Massey triple product [2].

If we wish to model magnetic flux tubes and magnetic fields of the coronal plasma loops then the linking number can be replaced with magnetic helicity $(\mathbb{H})$ that averages the linking of all pairs of field lines.

For a straight flux tube of flux $\Phi$, the helicity $\mathbb{H}$ is

$$
\mathbb{H}=T \Phi^{2} .
$$




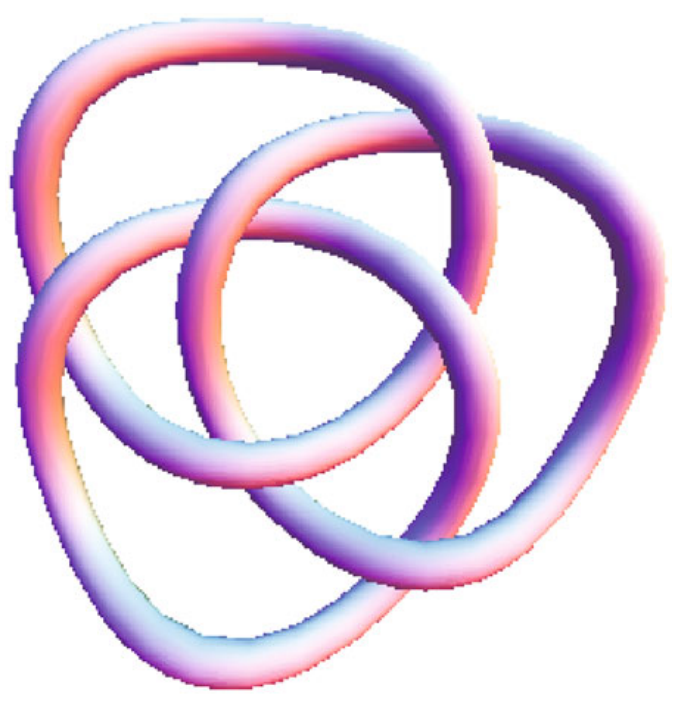

Fig. 6. Borromean ring knot.

The twist $T$ measures how much the helicity is generated by parallel currents. If $\mathrm{s}$ is the arc length along the central field line, then

$$
\frac{d T}{d s}=\frac{\mu_{0} J_{\|}}{4 \pi B_{\|}} .
$$

The writh $W$ for a curve with endpoints, i.e. how it kinks and coils, is defined by the Călugăreanu [5] formula,

$$
\mathbb{H}=(T+W) \Phi^{2} .
$$

Since the corona is nearly a conducting medium, the helicity is nearly conserved. It means that some twist $T$ can be converted into wirth $W$. However, not too much before a kink instability occurs and the CME takes place (Fig. 3).

\section{Cycles and topology}

The geometric figure that describes the solar cycles is analyzed with a 6 real dimensional Lorenz equation system.

Knots solutions of Lorenz systems have been found [4]. Here we will carry out an ergordic approach and make use of Poincaré maps.

\subsection{Complex generalization of the Lorenz equation}

The solar magnetic state is modelled with a complex generalization of the Lorenz equation [17,20]:

$$
\begin{aligned}
& \dot{A}=2 D B-A, \\
& \dot{B}=i A-\frac{1}{2} i \Omega A^{*}-B, \\
& \dot{\Omega}=-i A B-v \Omega .
\end{aligned}
$$

The dynamo number $D$ is given by:

$$
D=\alpha_{0} \triangle \Omega_{0} r_{\odot}^{3} / \eta_{t}^{2}
$$


Table 1. Estimating the dimension of the attractor of (7).

\begin{tabular}{|c|c|c|c|}
\hline$n$ & $N$ & $n$ & $N$ \\
\hline 9 & 55 & 125 & 1800 \\
\hline 16 & 107 & 243 & 6928 \\
\hline 25 & 169 & 256 & 637 \\
\hline 27 & 187 & 625 & 62895 \\
\hline 64 & 644 & 1024 & 241900 \\
\hline 81 & 911 & & \\
\hline
\end{tabular}

Here $\alpha$ is explicitly mentioned. [11] also include magnetic helicity and herewith $\alpha_{m}$. The dynamo number measures the strength of the two induction effects $\alpha_{0}$ and $\Delta \Omega_{0}$, relative to the diffusivity.

We studied the Poincaré map $(P(U))$ of the flow returning to the hyperplane $\operatorname{Re}(A)=0$, for the parameters $D=2$ and $v=0.4$, using the Runge-Kutta method. Since this flow is in 6 real dimensions (3 complex dimensions), the analysis of this Poincare map is much more numerically involved than that of the system of [19]. Starting with a point $(A, B, \Omega)$ we calculated a big number of returns to the hyperplane $\operatorname{Re}(A)=0$. The returns were situated in a 5 -dimensional box of size $4.1 \times 3.4 \times 4.0 \times 3.0 \times 6.0$. This box was divided into a grid of $n^{5}$ boxes. The aim was to find a subset of these boxes such that any point in any of the boxes returns to some of the boxes. If such a subset is found, then we let $U$ be the interior of the union of the boxes. We then found an attractor $\Lambda$ satisfying,

$$
\Lambda=\operatorname{closure} \bigcap_{n=0}^{\infty} P^{n}(U) .
$$

However we cannot numerically check the return of any point in the boxes, so instead we checked the returns of $m^{5}$ uniformly distributed points in each of the boxes. Some of these points did not return to the boxes, so instead we added new boxes containing these returns. For these new boxes, we again checked the returns, and this process was repeated several times. This process eventually stopped, and no more boxes were needed. Hence this is a numerical evidence for the existence of an attractor. These calculations were carried out for $m=4$ and $n=256$, and smaller values of these parameters. Each case resulted in that a trapping region was found.

\subsection{A chaotic attractor}

To estimate the dimension of the attractor, we estimated the attractor with a big number of returns of one point and checked how many boxes from the above mentioned grid that were needed to cover the returns. This was done with different numbers of returns, and different grid sizes.

If the grid consists of $n^{5}$ boxes, then it is expected that asymptotically $n^{s}$ boxes are needed to cover the attractor. If we let $N$ denote the number of boxes in the cover, by plotting $\log N$ against $\log n$ and fitting a straight line to the data, the slope of this line is an estimate of the dimension of the attractor. Table 1 shows the results from an estimate of the attractor using $10^{8}$ points. There is a plot of this in Fig. 6. The slope of the line suggests a dimension of the attractor (as a subset of the hyperplane) of about 1.7. Hence this suggests that the dimension of the attractor of the flow is about 2.7 [17].

\subsection{Cycle length and strength}

We studied the relationship between the period $n$ and period $n+1$. In both cases $v<0.4$.

When $D$ is sufficiently large and $v<1$, then the periodic solution becomes unstable, first by multiply periodic and finally by chaotic solutions. The periods are distributed in intervals.

When $D$ is increased shorter periods exist and also interesting, pronounced comet-like tail structures. Figure 8 shows when $D$ was set to 9.0. These tails illustrate how long periods are followed by short periods and vice versa. 


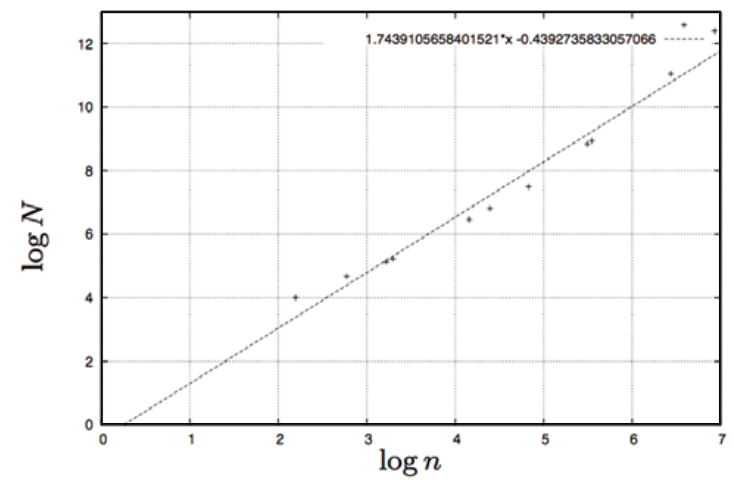

Fig. 7. Plot of the attractor dimension.

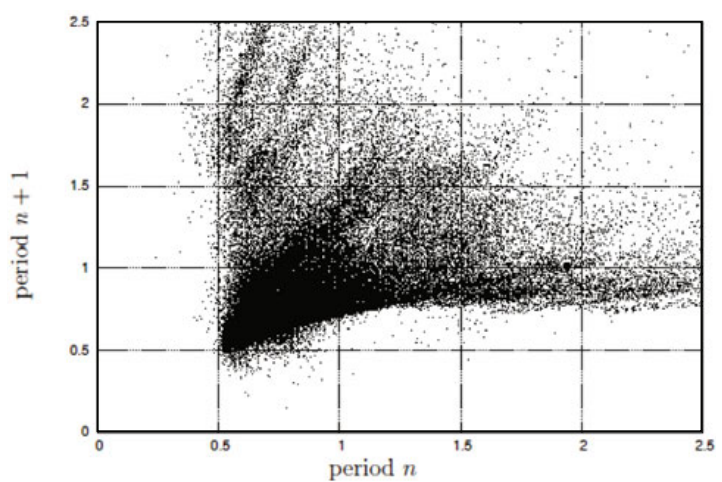

Fig. 8. Period $n$ vs. $n+1$ for $D=9.0$.

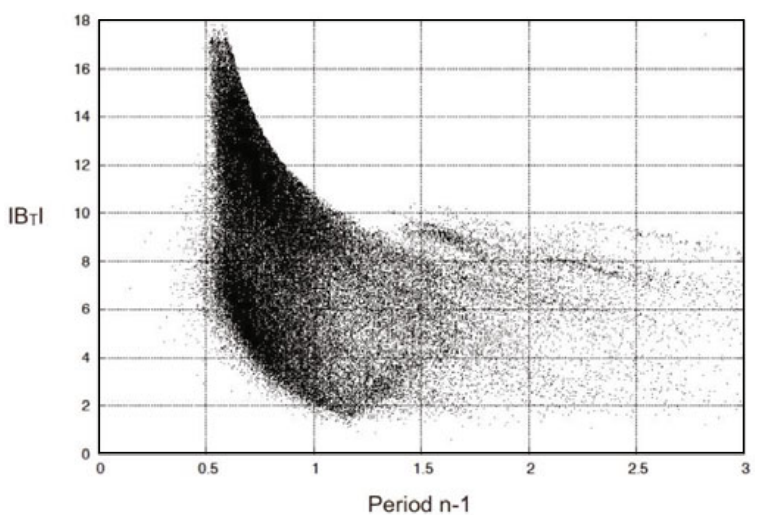

Fig. 9. Period $n-1$ vs. $\left|B_{T}\right|$ for $D=9.0$.

In Fig. 9 the absoute value of the toroidal field $\left|B_{T}\right|$ is plotted vs. the previous solar periods length. It is clear that a shorter period is followed by a more intense next cycle. time.

In Fig. $10 \operatorname{Re}\left(B_{T}\right)$ is plotted vs. time. Maunder minimum like periods are seen to appear along the 


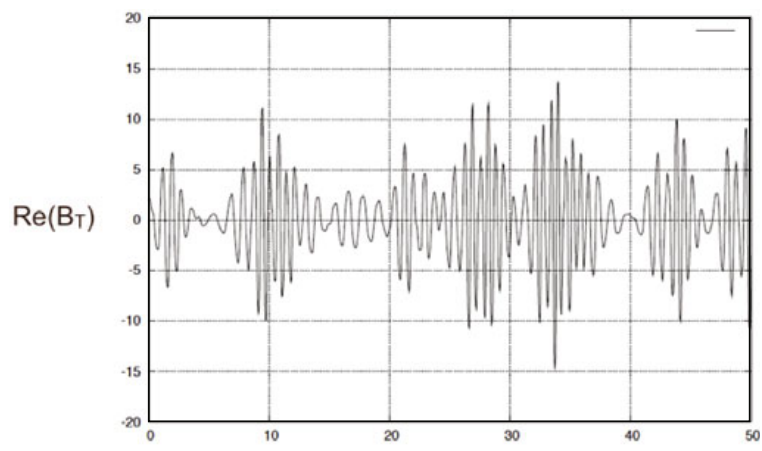

Fig. 10. $\operatorname{Re}\left(B_{T}\right)$ vs. time for $D=9.0$.

\section{Conclusions and future plans}

Both solar storms and solar cycles were described using topological methods.

The building blocks of solar storms, coronal plasma loops, were described with braids and knot theory. Eruption of prominences and CMEs were described using the conserving of helicity and conversion of twist into writh.

We used Poincaré maps to explore a dynamical system: a six-dimensional complex generalization [20].

The Poincaré maps gave numerical evidence that the flow has an attractor with fractal structure. The period was defined as the time needed for a point on a hyperplane to return the hyperplane again. The periods were distributed in an interval. For large values of the Dynamo number there is a long tail toward long periods and also other interesting features. These results can be compared with what [6] found. They emphasize that the velocity of the meridional flow is a critical factor in determining the period of the dynamo cycle. When introducing a random variation of the meridional velocity, they also found that a long cycle followed a short one.

We also found a relationship between a short cycle and a strong following cycle. Maunder minimalike cycles appears when time series of the toroidal field is studied.

We expect upcoming helioseismic observations with Solar Dynamics Observatory (SDO) [10] will give us improved physical values for the dynamical systems. More advanced equation systems including terms describing helicity will also be explored.

Finally, the interesting found structures will be further studied and neural networks will be used to learn these patterns for predictions $[15,16]$.

\section{References}

1. J. Beer, S. Tobias, N. Weiss, An Active Sun Throughout the Maunder Minimum, Solar Phys., 181, 237-249, 1998.

2. M.A. Berger, Third-order link integrals, J. Phys. A: Math. Gen. 23, 2787-2793, (1990).

3. M.A. Berger, Coronal Heating by Dissapation of magnetic Structure, Space Sc. Rev. 68, 3-14, (1994).

4. J.S. Birman, R.F. Williams, Knotted periodic orbits in dynamical systems - I: Lorenz's equations, Topology, Vol. 22, No. 1 47-82, (1983).

5. G. Călugăreanu, Sur les classes d'isotopie des noeds tridimensionels et leurs invariants, Czechoslovak Math. J, 11, 588-625, (1959).

6. M. Dikpati, P. Charbonneau, Astrophys. J., 518, (1999).

7. T. Frankel, The Geometry of Physics - An Introduction, second edition, Cambridge Univ. Press, (2007). 
8. P. Frick, D. Galyagin, D.V. Hoyt, E. Nesme-Ribes, K.H. Schatten, D. Sokoloff, V. Zakharov, Wavelet analysis of solar activity recorded by sunspot groups, Astron. Astrophys., 328, 670-681, (1997).

9. C.F. Gauss, Werke, Band 5, Königliche Gesellschaft der Wissenschaften, Göttingen, 602, (1867).

10. J.T. Hoeksema, the HMI Magnetic Team. The HMI Magnetic Field Measurement Program, presentation at SDO Team Meeting, March 25-28, Napa, California, (2008).

11. I. Kitiashvili, A.G. Kosovichev Ap. J., 688, L49-L52, (2008).

12. M.F. Knudsen, P. Riisager, B.H. Jacobsen, R. Muscheler, I. Snowball, M.-S. Seidenkrantz, Taking the pulse of the Sun during the Holocene by joint analysis of ${ }^{14} \mathrm{C}$ and ${ }^{10} \mathrm{Be}$, Geophys., Res., Lett., 36, L16701, (2009).

13. B.C. Low, Magnetic Energy and Helicity in Open Systems, in Magnetic Helicity in Space and Laboratory Plasmas, Editors M.R. Brown, R.C Canfield and A.A. Pevtsov, AGU Monograph 111, (1999).

14. H. Lundstedt, L. Liszka, R. Lundin, R. Muscheler, Long-term solar activity explored with wavelet methods, Ann. Geophys., 24, 1-9, (2006).

15. H. Lundstedt, Progress in space weather predictions and applications, Adv. Space Res., 36, 2516-2523, (2005a).

16. H. Lundstedt, Solar magnetic Activity: Topology and Predictions, doi.org/1072478/ s11600-0080062-6, Acta Geophysica, (2008).

17. H. Lundstedt,T. Persson, Modelling solar cycle length based on PoincarŽ maps for Lorenz-type equations, Ann. Geophys., 28, 993-1002, (2010).

18. K. Murasugi, Knot Theory and Its Applications, Modern Birhauser Classics, (2008).

19. S. Tobias, N.O. Weiss, V. Kirk, Chaotically modulated stellar dynamos, Mon. Not. R. Astron. Soc., 273, 1150-1166, (1995).

20. N.O. Weiss, F. Cattaneo, C.A. Jones, Geophys. Astrophys. Fluid Dyn. 30, 305, (1984). 